\title{
Commentary: Bilateral Ballism Following Streptococcal Infection, Associated with Psychiatric Disorder and Purpura
}

\author{
Antonio N Gomez-Valdes* \\ Department of Internal Medicine, Havana University School of Medicine, Havana, Cuba
}

Article Info

\section{Article Notes}

Received: December 6, 2018

Accepted: March 6, 2019

\section{*Correspondence:}

Antonio N Gomez-Valdes, Department of Internal Medicine, Havana University School of Medicine, Havana, Cuba; Email: antoniongomez@aol.com.

(c) 2019 Gomez-Valdes AN. This article is distributed under the terms of the Creative Commons Attribution 4.0 International License.

\section{Original Article Citation:}

BMJ Case Reports 2011; doi: 10.1136/bcr.05.2011.4261.
In the case reported of our article entitled "Bilateral ballism followed streptococcal infection associated with psychiatric disorder and purpura "based on the clinical judgment it was possible to associate the three main clinical manifestation (movement disorder, psychiatric disorder and purpura) under the same etiologic diagnosis that was streptococcal infection of the pharynx confirmed fully. Indeed, in the diagnostic evaluation we try always to be unique when it is reasonably possible. The target treatment removing the infectious focus by tonsillectomy associated with the administration of the specific antibiotic followed by significant improvement of three clinical manifestations especially the biballism confirming the diagnostic pose above mentioned.

The retrospective analysis of the study of the patient reported allow us to consider the following: This patient at teenager was not treated fully of the primary streptococcal infection of the pharynx and of secondary prevention of recurrence, limiting the use of penicillin for each episode of pharyngeal infection with a palliative but not curative effect during the patient's life. Therefore, the infectious process led to chronicity with latent infection causing the last outbreak in the patient's adult with the clinical form of rheumatic fever at this age, though with some peculiar modification. Thus, we can infer that biballism might have been a Sydenham's chorea caused by the first streptococcal infection during the childhood of our patient, though some choreoathetoid movements were accompanying to bilateral ballism. The arthritis was monoarticular as rheumatic fever in adult. The depression depended from the organic process according to psychiatric diagnosis. The purpura was also linked to the central immune-infectious disease having rule out other causes. There was no carditis that is most frequent in children and adolescents but may be absent in the adult as our patient. In conclusion, the wrong medical and therapeutic management of this patient at the beginning of the illness and subsequently resulted in a long evolution of the process that led to the development of the rheumatic fever into adulthood. One of the anatomo-clinical features of this disease is the biballism following a streptococcal infection that is extremely rare even more in the orbit of the rheumatic fever. Our clinical research arises during the medical practice with the purpose of to attain the best diagnosis and treatment for the benefit of the patients, avoiding always any damage to their physical and mental integrity. A clinical observation can be the beginning of a discovery. This is the phenomenon according to Claude Bernard. Claude Bernard was born in France in the year 1813 and is considered one of the greatest minds of science. His biography can be found in 
all libraries across the world. During the medical practice and specifically from the diagnostic discussion can arises of new idea on a topic based on the clinical observation.
This clinical research and its result must be always rigged with the benefit to the patient avoiding any damage to the physical and mental integrity of the patient. 